\title{
Género como factor asociado a sufrir determinado tipo de violencia
}

\author{
Cristina Haydée Arrom Suhurt ${ }^{\mathrm{I}}$, Maria del Pilar FrescoI, Carmen Marina Arrom ${ }^{\mathrm{II}}$, Marcos Capurro ${ }^{\mathrm{I}}$, \\ Margarita Samudio $^{\mathrm{III}}$, Maria Auxiliadora Arrom ${ }^{\mathrm{II}}$, Andres Claudelino Arce ${ }^{\mathrm{I}}$, Mirta Romero \\ I. Cátedra de Psiquiatría-Facultad de Ciencias Médicas de la Universidad Nacional de Asunción, Paraguay. \\ II Centro para el Desarrollo de la Investigación Científica CEDIC/Díaz Gill, Paraguay \\ III. Instituto de Investigaciones en Ciencias de la Salud-Universidad Nacional de Asunción (IICS-UNA), \\ Paraguay
}

Cómo referenciar este artículo/ How to reference this article:
Arrom C, Fresco MP, Arrom CM, Capurro M, Samudio M, Arrom MA, et al. Género como factor asociado a sufrir determinado tipo de violencia. Mem. Inst. Investig. Cienc. Salud. 2015;13(3)51-57

\section{RE S U M E N}

La violencia es una forma de relación social que produce un profundo daño en el desarrollo psicosocial de las personas, en la expresión de sus múltiples capacidades y salud mental integral. El objetivo del estudio es caracterizar la violencia de género en pacientes adultos y adultas del servicio ambulatorio de la Cátedra de Psiquiatría de la Facultad de Ciencias Médicas. Estudio trasversal, descriptivo en el que se realizó la revisión retrospectiva de 254 fichas clínicas de pacientes atendidos para psicoterapia entre 2010 y 2013. La mayoría de los pacientes $70,1 \%(n=178)$ era de sexo femenino; $48 \%$ $(n=121)$ proveniente de la capital; $65,3 \%(n=166)$ de estado civil soltero; $37 \%(n=93)$ tenía entre 19 a 25 años y $29 \%(n=73) 26$ a 35 años; $27,5 \%(n=70)$ sufrió violencia psicológica, 5,5\% $(n=11)$ violencia física, $21 \%(n=54)$ psicológica y física, 3,5\% $(n=9)$ abuso sexual, $10,6 \%(n=27)$ abuso sexual, violencia física y psicológica, $3,1 \%(8)$ abuso sexual y violencia psicológica, $2,4 \%(n=6)$ otras combinaciones. Se encontró asociación entre ser mujer y víctima de abuso $(p=0,0011)$ y violencia parental $(p=0,02)$ y ser varón con violencia fuera del hogar $(p=0,008)$ y una tendencia a sufrir violencia por abandono $o$ negligencia. Se encontraron distintas formas de violencia padecida por los hombres y mujeres consultantes. Hubo una clara violencia de género en mujeres víctimas de abuso, y en varones violencia fuera del ámbito doméstico.

Palabras clave: violencia intrafamiliar-pacientes-salud mental.

\section{Gender as an associated factor for suffering certain types of violence}

\section{A B S T R A C T}

Violence is a form of social relation causing a deep damage in the psycho-social development of people in the expression of their multiple capacities and comprehensive mental health. The objective was to characterize gender violence in adult male and female patients of the outpatient service of the Department of Psychiatry.: Cross-sectional descriptive study that analyzed 254 clinical records of patients that received psychotherapy care between 2010 and 2013. Most patients were female, $70.1 \%$ (178); from the capital, 48\% (121); single 65.3\% (166); 37\% (93) was between 19 to 25 years old and $29 \%$ (73) between 26 to 35 years, $27.5 \%$ (70) suffered psychological violence, $5.5 \%$ (11) physical violence, $21 \%$ (54) psychological and physical violence, $3.5 \%$ (9) sexual abuse, $10.6 \%$ (27) sexual abuse, physical and psychological violence, $3.1 \%$ (8) sexual abuse and psychological violence, $2.4 \%$ (6) others combinations, $5.1 \%$ (13) no type of violence, $22 \%$ (56) without information at the time of the study. There was 
association between being a woman and abuse victim $(p=0,0011)$ and parent violence $(p=0,02)$ and a trend between being a man and suffering neglect $(p=0.008)$.: Male and female patients suffered different forms of violence. There was clear gender violence in female victims of abuse and in men violence outside of the domestic environment.

Keywords: intrafamily violence-patients-mental health.

\section{INTRODUCCIÓN}

El género determina el poder diferencial y el control que hombres y mujeres tienen sobre los determinantes socio-económicos de sus vidas y salud mental, la posición y condición social, la forma en que la sociedad los trata y su susceptibilidad y exposición a riesgos específicos para la salud mental (1).

La Organización de las Naciones Unidas define la violencia contra la mujer como todo acto de violencia de género que resulte o pueda tener como resultado un daño físico, sexual o psicológico para la mujer, inclusive las amenazas de tales actos, la coacción o la privación arbitraria de libertad, tanto si se producen en la vida pública como en la privada (2). Cifras recientes de prevalencia a nivel mundial muestran que el $35 \%$ de las mujeres en el mundo ha sufrido violencia de pareja o violencia sexual por terceros en algún momento de su vida (2).

En cuanto a factores de riesgo de ser víctimas de violencia de pareja o de violencia sexual se encuentran el tener un bajo nivel de instrucción, haber presenciado actos de violencia doméstica entre los padres, haber estado expuestos a maltratos durante la infancia, actitudes de aceptación de la violencia y las desigualdades de género entre otros (2).

Estudios internacionales dan cuenta que la violencia de pareja y la violencia sexual son perpetradas mayormente por hombres contra mujeres y niñas, y aproximadamente el $20 \%$ de las mujeres y el $5 \%-10 \%$ de los hombres refieren haber sido víctimas de abuso sexual en la infancia (2). Un estudio en 304 puérperas en un Hospital de Asunción puso en evidencia que $75 \%$ había sufrido violencia psicológica, $63 \%$ violencia física y $8,6 \%$ abuso sexual de parte de su pareja íntima con una asociación significativa entre la exposición a estos tipos de violencia y padecimiento de episodios depresivos leves, moderados y graves.(3). Otra investigación en 100 mujeres de un Hospital de Lambaré evidenció que $8 \%$ había sido forzada a tener relaciones con su pareja, en tanto que otro $6 \%$ sufrió intento de abuso (4).

En Paraguay las actitudes y los comportamientos de la población muestran que la violencia hacia las mujeres aún hoy todavía se justifica y se encuentra arraigada como creencia de que es un problema del ámbito privado en el marco del cual agresores tienen impunidad (5). Si bien la participación de las mujeres en el mundo laboral avanzó, las brechas salariales de género continúan en la mayoría de los países del mundo (4) y en Paraguay ésta aumentó entre varones y mujeres de $4 \%$ a $7 \%$, según un estudio publicado por el Banco Interamericano de Desarrollo (6) lo que genera mayor desigualdad y vulnera la situación de la violencia de género.

La falta de acceso a la justicia en casos de violencia de género, doméstica e intrafamiliar constituye otro factor que se suma a la impunidad imperante en Paraguay con las grandes desigualdades que existen, sociales y económicas, donde la justicia funciona para quien cuenta con medios económicos para demandar y denunciar la violencia (5).

Se requiere aumentar las competencias de los profesionales de la salud para reconocer y tratar las consecuencias que la violencia doméstica, los abusos sexuales y el estrés agudo y crónico que padecen las mujeres por razones de género (1). El objetivo del estudio es caracterizar la violencia de género en pacientes adultos del servicio ambulatorio de la Cátedra de Psiquiatría de la Facultad de Ciencias Médicas.

\section{PACIENTES Y MÉTODO}

El estudio fue trasversal, de tipo descriptivo. Fueron analizadas 254 fichas clínicas de pacientes adultos (de 19 y más años) remitidos a Psicoterapia en el Servicio Ambulatorio de enero del 2010 a marzo del 2013. Las fichas fueron completadas por Profesionales Médicos Residentes y Psicólogas del Servicio.

Para los fines del estudio que tuvo como propósito la incidencia de la distribución de violencia por razones de género en una población de personas adultas con antecedentes de violencia dentro o fuera del ámbito familiar, se establecieron como variables de estudio, 
datos sociodemográficos (edad, sexo, procedencia); impresiones diagnósticas en el Eje I y Eje II configuradas en base a criterios propuestos por el Manual Diagnóstico y Estadístico (DSM IV TR) de la Asociación Americana de Psiquiatría. Conceptos de: violencia física utilizando la clasificación de UNICEF para Violencia Doméstica contra Mujeres y Niñas (7) que la define como "malos tratos físicos tales como bofetadas, golpes, torsión de brazos, puñaladas, estrangulación, quemaduras, sofocación, patadas, amenazas con armas u otros objetos, y en casos extremos el asesinato"; violencia psicológica o emocional, como "intimidar, atormentar a la víctima y asume diferentes formas: amenazas de abandono o abuso, reclusión en el hogar, vigilancia estricta, amenazas de destitución del cuidado de los hijos, destrucción de objetos, aislamiento, agresiones verbales y humillaciones constantes"; abuso sexual que se define como "relaciones sexuales forzadas, impuestas mediante amenazas e intimidaciones o con la fuerza física, la coerción a prácticas sexuales indeseadas, o la constricción a tener relaciones sexuales con terceros"; (7) negligencia o abandono como "falta de protección y cuidados físicos al niño de parte de sus figuras de custodia, así como el abandono de sus necesidades evolutivas por falta de estimulación cognitivas que no se deben a carencias extremas de recursos socioeconómicos" (7). También se consignaron datos del contexto en el cual se produjo la violencia.

Del total de 254 pacientes, 203 tenían información referente a exposición a violencia, de los cuales $190(93,6 \%)$ refirieron haber estado expuestos a violencia en diferentes ámbitos (intra y extra familiar) mientras que $13(6,4 \%)$ no. Se procedió a analizar en las 203 personas la manera en que incidió la violencia y sus diferentes formas, según recayera en la condición de ser hombre o mujer dentro del grupo.

Los datos fueron cargados en una hoja de cálculo Excel y posteriormente analizados en el paquete estadístico SPSS Versión 15.0 para Windows 7. Los resultados se expresaron en forma de frecuencia absoluta y relativa y para indagar posibles asociaciones entre tipo de violencia y género se utilizó la prueba de chi cuadrado a un nivel de significancia de 0,05.

\section{RESULTADOS}

La mayoría de los consultantes $70,1 \%(n=178)$ fue de sexo femenino. El $37 \%(n=93)$ de la población que consultó tenía entre 19 a 25 años. El mayor porcentaje 48\% $(n=121)$ provenía de la Capital; $14,6 \%(n=37)$ del departamento Central, el $65,3 \%$ $(n=166)$ era de estado civil soltero. (Tabla 1$)$ 
Tabla 1. Características de los pacientes atendidos para psicoterapia. $n=254$

\begin{tabular}{|c|c|c|}
\hline Características & $\mathbf{n}$ & $\%$ \\
\hline \multicolumn{3}{|l|}{ Sexo } \\
\hline Mujer & 178 & 70,1 \\
\hline Varón & 76 & 29,9 \\
\hline \multicolumn{3}{|l|}{ Rango de Edad (año) } \\
\hline 19 a 25 & 93 & 36,6 \\
\hline 26 a 35 & 73 & 28,7 \\
\hline 36 a 45 & 35 & 13,8 \\
\hline 46 a 55 & 37 & 14,6 \\
\hline$>55$ & 15 & 5,9 \\
\hline Desconocido & 1 & 0,4 \\
\hline \multicolumn{3}{|l|}{ Procedencia } \\
\hline Capital & 121 & 47,6 \\
\hline Central & 37 & 14,6 \\
\hline Interior & 75 & 29,5 \\
\hline Exterior & 11 & 4,3 \\
\hline Desconocida & 10 & 3,9 \\
\hline \multicolumn{3}{|l|}{ Estado Civil } \\
\hline Soltero & 166 & 65,3 \\
\hline En pareja/casado & 52 & 20,5 \\
\hline Divorciado/separado & 9 & 3,5 \\
\hline Otros & 2 & 0,8 \\
\hline Desconocido & 25 & 10 \\
\hline
\end{tabular}

En relación al tipo de violencia que sufrieron los pacientes, se pudo observar que el $27,6 \%(n=70)$ sufrió violencia psicológica; $21,3 \% \quad(n=54)$ psicológica y física; 10,6\% $(\mathrm{n}=27)$ psicológica, física y abuso sexual entre los más frecuentes. (Tabla 2 ) 
Tabla 2. Tipo de violencia en los pacientes atendidos para psicoterapia. $n=254$

\begin{tabular}{lll}
\hline Tipo de Violencia & $\mathbf{n}$ & $\mathbf{\%}$ \\
\hline Psicológica & 70 & 27,6 \\
Psicológica y física & 54 & 21,3 \\
Psicológica, física, abuso sexual & 27 & 10,6 \\
Física & 11 & 4,3 \\
Abuso sexual & 9 & 3,5 \\
Abuso sexual y psicológica & 8 & 3,1 \\
Otras combinaciones & 6 & 2,4 \\
No sufrió violencia & 13 & 5,1 \\
Desconocido & 56 & 22 \\
\hline
\end{tabular}

Las mujeres presentaron significativamente mayor frecuencia de abuso sexual (valor $p=0.00119$ ) y violencia parental (valor $p=0,02$ ) que los varones. El abandono o negligencia fue más frecuente en los varones pero la diferencia no llegó a significancia estadística (valor $p=0,08$ ). Ser víctima de violencia fuera del hogar estuvo relacionado con el sexo masculino (valor $p=0.004$ ). (Tabla 3)

Tabla 3. Asociación entre sexo y ser víctima de violencia. $n=198$

\begin{tabular}{llll}
\hline Tipo de violencia & Femenino & Masculino & \\
& $\mathbf{n = 1 5 0}$ & $\mathbf{n = 4 8}$ & Valor $\mathbf{p ~}$ \\
\hline Abuso sexual & $42(28,0)$ & $2(4,2)$ & 0,0011 \\
Abandono (negligencia) & $1(0,7)$ & $2(4,2)$ & 0,08 \\
Violencia parental & $55(36,7)$ & $27(77,1)$ & 0,02 \\
Violencia fuera del hogar & $9(6)$ & $8(16,7)$ & 0,004 \\
\hline
\end{tabular}

\section{DISCUSIÓN}

Casi la totalidad de la población de estudio, mayoritariamente femenina y menor de 35 años, manifestó haber padecido distintas formas de violencia durante su infancia, adolescencia y adultez por parte de su familia, pareja íntima, y otros actores, hallazgos que coinciden con estudios de referencia como el de Multipaís de la OMS (8) que confirmó que la violencia infligida por la pareja está muy difundida en todos los países y la de pareja íntima aún continúa como una de las formas más comunes contra la mujer. Estudios existentes indican que a menudo coexisten diferentes formas de violencia y que la violencia física de parte de la pareja va acompañada a menudo de abuso sexual y generalmente de maltrato emocional (8) En el mismo estudio, entre $23 \%$ y $56 \%$ de las mujeres que habían sufrido alguna vez violencia física o sexual de sus parejas, informaron que habían padecido ambas formas (8-11).

La violencia de género expresada en el presente estudio se vinculó en forma significativa a las mujeres como víctimas de abuso sexual, de violencia doméstica por parte de su pareja, asociación que coincide con los estudios sobre la violencia de género $(2,3)$. Numerosos estudios ya han dado cuenta que la abrumadora carga mundial de violencia infligida por la pareja íntima es sobrellevada por las mujeres, aunque pudieran ellas ser violentas en sus relaciones con los hombres, a menudo esto se presenta en 
defensa propia. La evidencia de que los agresores más comunes en la violencia contra la mujer son sus compañeros íntimos de sexo masculino sigue vigente $(8,9,11,12)$.

Se constatan estereotipos de género culturalmente establecidos que siguen identificando o justificando que la violencia sufrida por las mujeres se da en su ámbito natural, el doméstico, en tanto que los hombres, acostumbrados a expectativas culturales de mayor libertad, la sufren fuera del mismo, según muestran los resultados de este estudio que vinculó significativamente esta evidencia. Es decir, como se enumeran en investigaciones de referencia, es mucho más probable que los hombres sean víctimas de violencia "en actos perpetrados por desconocidos o por conocidos que por alguien más cercano a ellos" (13).

Paraguay mantiene una amplia inequidad de género hoy en día (13), ya que decenas de mujeres mueren anualmente ante la insuficiencia del cumplimiento de las obligaciones estatales de proteger sus vidas, por la pobreza, exacerbada cultura patriarcal y religiosa, la falta de acceso a la justicia, la falta de educación integral de la sexualidad, la insuficiente promoción del acceso de las mujeres a los cargos electivos, la discriminación legal, la ausencia de una legislación adecuada para la protección efectiva de sus derechos y la extendida precariedad laboral de las trabajadoras domésticas (13).

Hay que resaltar que el grupo estudiado evidenció claro padecimiento de violencia por su condición de género (8), y se encuentra en un Servicio de un hospital escuela para tratamiento por distintos trastornos en su salud mental. Las consecuencias de la violencia pueden persistir mucho tiempo después de que haya cesado la misma y cuando mayor gravedad reviste el maltrato, mayores serán las repercusiones en la salud física y mental de la mujer y de sus hijos e hijas en caso de que fueran testigos ante tales situaciones, como lo indican múltiples evidencias científicas en la región de América Latina y en Paraguay $(8,9,11,14)$.

La psicoeducación en prevención de la violencia de género (15) podría contribuir a paliar el dolor y sufrimientos mentales de pacientes y su entorno familiar en Servicios de Salud Mental como el Ambulatorio de la Cátedra de Psiquiatría de la Universidad Nacional de Asunción (16) además de acciones de intervención comunitaria, un adecuado abordaje en instituciones educativas sobre la violencia, la cultura machista vigente y la impunidad y sus consecuencias en salud mental $(15,17)$. La violencia de género se debería combatir con cambios culturales y acceso a la justicia en Paraguay y en la mayoría de los países de América Latina como lo recomiendan organismos internacionales $(5,8)$.

Las normas sociales y culturales relacionadas al género constituyen pautas 0 "expectativas de comportamiento" (9) que reglamentan roles y relaciones entre hombres y mujeres dentro de un grupo cultural y muchas veces hacen a las mujeres vulnerables a la violencia infligida por la pareja imponiéndoles mayor riesgo y aprobando o favoreciendo la aceptabilidad de la violencia. Encaminar esfuerzos por modificar estas constituye un elemento primordial en la prevención primaria de estas formas de violencia. (9).

La violencia de género es producto de una socialización diferencial entre hombres y mujeres y además, fruto de procesos de legitimación social y cultural de la violencia. Algunas propuestas educativas incluyen desvincular la masculinidad patriarcal de la violencia de género con alternativas que utilicen resolución de conflictos, educación sentimental, cultura de paz y relaciones entre interculturalidad y violencia de género. (18).

\section{REFERENCIAS BIBLIOGRAFICAS}

1. Organización Mundial de la Salud (OMS) [Internet]. Washington, DC-USA: OMS; [citado 18 mayo de 2013]. Disponible en: www.caib.es/sacmicrofront/archivopub.do?ctrl $=$ MCRST456ZI93635

2. Organización Mundial de la Salud (OMS) [Internet]. Washington, DC-USA: OMS; [citado 18 mayo de 2013]. Disponible en: http://www.who.int/mediacentre/factsheets/fs 239/es/
3. Arrom C, Samudio M, Arrom CM, Lampert N, Arrom MA, Orué E. Violencia intrafamiliar, de pareja íntima y abuso sexual en puerperio inmediato. Su relación con depresión. Revista Paraguaya de Psiquiatría. 2013;1(2):17-23.

4. Galeano $A$, Orrego $B$, Pérez $M$, Ruiz V, Arrom MA, Ruoti $M$, et al. Violencia intrafamiliar sufrida por mujeres en un Hospital Público. Revista Paraguaya de Psiquiatría. 2015;3(1):42-9. 
5. González Vera, M. La violencia de género se combate con cambios culturales y acceso a la justicia en: Codehupy (2013) Yvypóra Derécho Paraguáipe. Derechos humanos en Paraguay 2012. Asunción: Codehupy pp. 2013; 451-63.

6. Banco Interamericano de Desarrollo (BID) [Internet]. Washington, DC-USA: Banco Interamericano de Desarrollo; [citado 18 mayo de 2013]. Disponible en: http://www.iadb.org/es/noticias/articulos/201 2-10-15/diferencia-salarial-entre-hombres-ymujeres, 10155.html

7. UNICEF [Internet]. Florencia-Italia: Innocenti Digest-Unicef; [citado 2 abril 2015]. Disponible en: http://www.unicefirc.org/publications/pdf/digest6s.pdf

8. Organización Mundial de la Salud-Oficina Regional para las Américas-Organización Panamericana de la Salud [Internet]. Washington D.C., EEUU: OPS-OMS; [citado 2 abril 2014]. Disponible en: http://apps.who.int/iris/bitstream/10665/988 16/1/WHO_RHR_12.36_spa.pdf?ua =1

9. Organización Mundial de la Salud-Escuela de Higiene y Medicina Tropical de Londres. [Internet]. Washington D.C.: OPS-OMS; [citado 10 Mayo 2014]. Disponible en: http://apps.who.int/iris/bitstream/10665/448 10/1/9789275316351_spa.pdf

10. Arrom C, Lampert N, Godoy N, Zarza Z, Arrom CM. Percepciones de adolescentes puérperas sobre el comportamiento de mujeres víctimas de violencia doméstica. Revista Paraguaya de Psiquiatría. 2015;3(1):20-5.

11. Brendel C, Heikel MA [Internet]. Los costos empresariales de la violencia contra las mujeres en Paraguay. Cooperación AlemanaGIZ. [citado 16 de setiembre de 2015]. Disponible en PDF:

http://info.comvomujer.org.pe/catalogocomvo /productoscatalogos_3_2015/ComVoMujer_Re sumendelestudiodecostosPY_2015.pdfCEDAW
12. Organización Mundial de la Salud [Internet]. Ginebra-Suiza: OMS; [citado 12 de agosto de 2013]. Disponible en: http://www.who.int/mediacentre/factsheets/fs 239/es/

13. ONU MUJERES-Centro Paraguayo de Estudios de Población [Internet]. AsunciónParaguay: ONU MUJERES-CEPEP. [citado 20 mayo 2015]. Disponible en: http://www.cepep.org.py/archivos/NUEVOS_A PORTES_ESTUDIO_VIOLENCIA_CONTRA_LA_ MUJER.pdf

14. Fresco MP, Arrom $C$, Samudio $M$, Arrom CM, Capurro $M$, Arrom MA, et al. Psicopatología en adultos con antecedentes de violencia intrafamiliar. Revista Paraguaya de Psiquiatría. 2015;3(1):8-12.

15. González Vera, M. Patriarcado, machismo e impunidad: causas de la cotidiana violencia de género En: Coordinadora de Derechos Humanos del Paraguay (Codehupy). Derechos humanos en Paraguay/ Yvypóra Derécho Paraguáipe. Codehupy. Asunción: Mercurio S.A.; 2012:447-63.

16. Secretaría de la Mujer. Plan Nacional de Igualdad de Oportunidades entre hombres y mujeres. Presidencia de la República del Paraguay. Fondo de Población de las Naciones Unidas. Asunción: Edit. Arte SA. 2008.

17. Instituto Interamericano de Derechos Humanos, Convención CEDAW y Protocolo Facultativo. Convención sobre la Eliminación de todas las formas de discriminación contra la mujer. Segunda edición. San José, Costa Rica: Instituto Interamericano de Derechos Humanos; 2004.

18. Barragan Medero F. Educación, adolescencia y violencia de género: Les amours finissent un jour. Tenerife, España: Universidad de La Laguna. 2006;6(1):31-53. 